\title{
Automated Attitude Sensor Calibration: Progress and Plans
}

\author{
Joseph Sedlak and Joseph Hashmall ${ }^{*}$ \\ a.i. solutions, Inc., Lanham MD 20706 USA
}

\begin{abstract}
This paper describes ongoing work at NASAGoddard Space Flight Center to improve the quality of spaceeraft attitude sensor calibration and reduce costs by automating parts of the calibration process. The new calibration software can autonomously preview data quality over a given time span, select a subset of the data for processing, perform the requested calibration, and outpat a report. This level of automation is currently being implemented for two specific applications: inertial reference wait (IRU) calibration and sensor aligament calibration. The IRU calibration utility makes use of a sequential version of the Davenport algorithm. This utility has been successfully tested with simulated and actual fight data. The alignment calibration is still in the early testing stage. Both utilities will be incorporated into the institutional attitude ground support system.
\end{abstract}

\section{Introduction}

Attitude sensor calibration is one of the critical tasks performed during the first few weeks of most space missions. It often is repeated later in a mission to maintain sensor accuracy. Calibration compensates attitude sensors for the effects of launch shock, mean temperature change, release of gravitational stress, and age-related changes. Depending on mission requirements, the calibration paraneters usually include primary sensor alignments and inertial reference unit (IRU) alignment, scale factors, and biases. Similar corrections may be determined for magnetometers and Sun and Earth sensors. Magnetometer calibration often includes magnetic torquer compensation. More rarely, it is useful to estimate improvements to the Sun sensor or star tracker transfer function that converts counts to angles in the sensor field of view.

Flight Dynamics personnel supporting missions for the NASA/Goddard Space Flight Center (GSFC) regularly provide calibration parameter estimates and related analyses for many Earth-orbiting and Lagrange point missions. The existing calibration software utilities are reliable and well-tested in the operations environment but do require input from an experienced analyst. The current work is aimed at adding a level of automation on top of these calibration utilities that will capture some of the analysts' procedures and experience with the data. It is expected that this will help control the cost of support, increase reliability, and simplify training for new personnel.

Section II discusses plans for a general automated calibration system that monitors the data in real-time, schedules the calibration (including attitude maneuvers, if needed), performs the calibration, and validates the results. Such a system could be developed for either ground-based or onboard use. However, the prototype software described in Sections III and IV implements only a portion of this general system. In particular, it does not include the expert system technology needed to make decisions about scheduling recalibration. What it does include is a ground-based system that is able to preview data quality over a given time span, select a subset of the data for processing, call the calibration utility, and report the results. This level of automation is currently being developed for two specific applications: IRU calibration and sensor alignment calibration. Progress in these areas is discussed in Sections III and IV. Section III describes the IRU calibration subsystem, which has been successfully tested for a variety of mission scenarios with simulated and actual flight data. Section IV describes the alignment calibration subsystem. Both subsystems are to be incorporated into the NASA/GSFC institutional attitude ground support system. ${ }^{1}$ This software is programmed using the Matiab ${ }^{\circ}$ computing language from The MathWorks, Inc. Section V gives some conclusions and directions for future work.

\section{Automated Calibration - High Level Design}

Attitude sensor calibration usually is performed during the first month for any mission with tight attitude accuracy tolerances. It is performed again, as needed, if significant sensor drift is detected. The process for performing the calibration normally requires several steps. A calibration plan is first prepared; this includes a request

\footnotetext{
- Senior Engineers, a.i. solutions, Inc., 10001 Derekwood Lane, Suite 215, Lanham, MD 20706 USA
} 
for a minimum data volume, the data type, and may specify special attitude maneuvers (i.e., slews). If maneuvers are requested, these must be scheduled around the previously established science and engineering timeline and must respect all mission constraints. The data are then processed to update the calibration parameters. The validity of the parameters is checked. Finally, a decision is made concerning whether to use the new parameters. Analysts must monitor the sensors and attitude to recognize problems when they occur and decide whether a recalibration would be useful.

A general automated system for attitude sensor calibration has been proposed in Ref. 2. That reference provides a general outline for the functional flow of the system and a detailed design and implementation for the IRU calibration subsystem. This section reviews the overall design, and the next sections discuss the continuing work on the IRU and atignment calibration subsystems.

The guiding principle when building an automated calibration system is that it should capture the techniques used by experienced analysts. These should be implemented as options in a software package that can be run either in a fully automated mode with preset parameters or a human-in-the-loop mode where choices can be made about the data selection. A complete calibration system needs to perform the following functions:

- Monitor data

- Request calibration when needed

- Plan data acquisition, including maneuvers

- Verify schedule

- Calibrate

- Validate

Figure 1 presents a high-level design for such a system. ${ }^{2}$ The proposed system design includes the functions listed above and allows for human intervention at a number of key decision points.

The first function is to monitor the sensor data. This function should look for sensor trends and noise levels. The attitude should be independently estimated and compared with the onboard estimate. The sensor residuals (observations minus predictions) should be monitored for any trends in the mean error or noise.

If it is found that systematic sensor errors exceed a certain tolerance, the system should request recalibration for that sensor. It is clear that this decision process could be made very sophisticated using artificial intelligence methods. At this time, such methods have not been investigated for this system. It remains up to the analyst to decide when to perform a new calibration.

When recalibration is needed, the proposed automated system should indicate the level of concern or importance for the errors in the affected sensor. This level is referred to as the Alert Level in Figure 1 . The system would call for human intervention if the Alert Level is very high. In general, the Alert Level would be used in planning the calibration. It would help determine how soon calibration is needed and whether the significance is high enough to interrupt the science schedule, if needed.

To plan the calibration, the system will request certain data types, data frequency, and data volume. For example, if magnetometer recalibration is needed, the system will request torquer data along with the magnetometer and other attitude sensor data. Different data frequencies may be available in the various telemetry modes that could be selected by the system. The data volume is a function of the total time span and frequency. The system must request time spans (with maneuvers, if needed) that satisfy the calibration requirements. If maneuvers are scheduled, it must verify that these satisfy all mission constraints.

Some calibrations require attitude maneuvers, such as IRU calibration and transfer function calibration. For example, the IRU calibration needs varying rates on all axes to make all the parameters observable. The sensor transfer function calibration needs measurements that thoroughly sample the sensor field of view.

It is difficult to automate maneuver planning. The maneuvers must slew the spacecraft over sufficiently large angles while respecting the constraints. There will also be other attitude requirements, such as ensuring that intermediate attitudes have enough guide stars and the trackers are not occulted. Each mission will have its own specific requirements. As an example, a utility has been created to aid in maneuver planning for the Rossi X-ray Timing Explorer (RXTE). This program has been used for several years to help plan maneuvers for IRU calibrations. The utility suggests sets of maneuvers and provides information to the analyst concerning the constraints and occultations. The analyst can vary the search parameters until a satisfactory set of maneuvers is found. This utility is representative of the type of planning tool that is needed. The current version is not sufficiently automated or general enough to apply to other missions, but it does provide a starting point for designing a generic maneuver planning tool. 


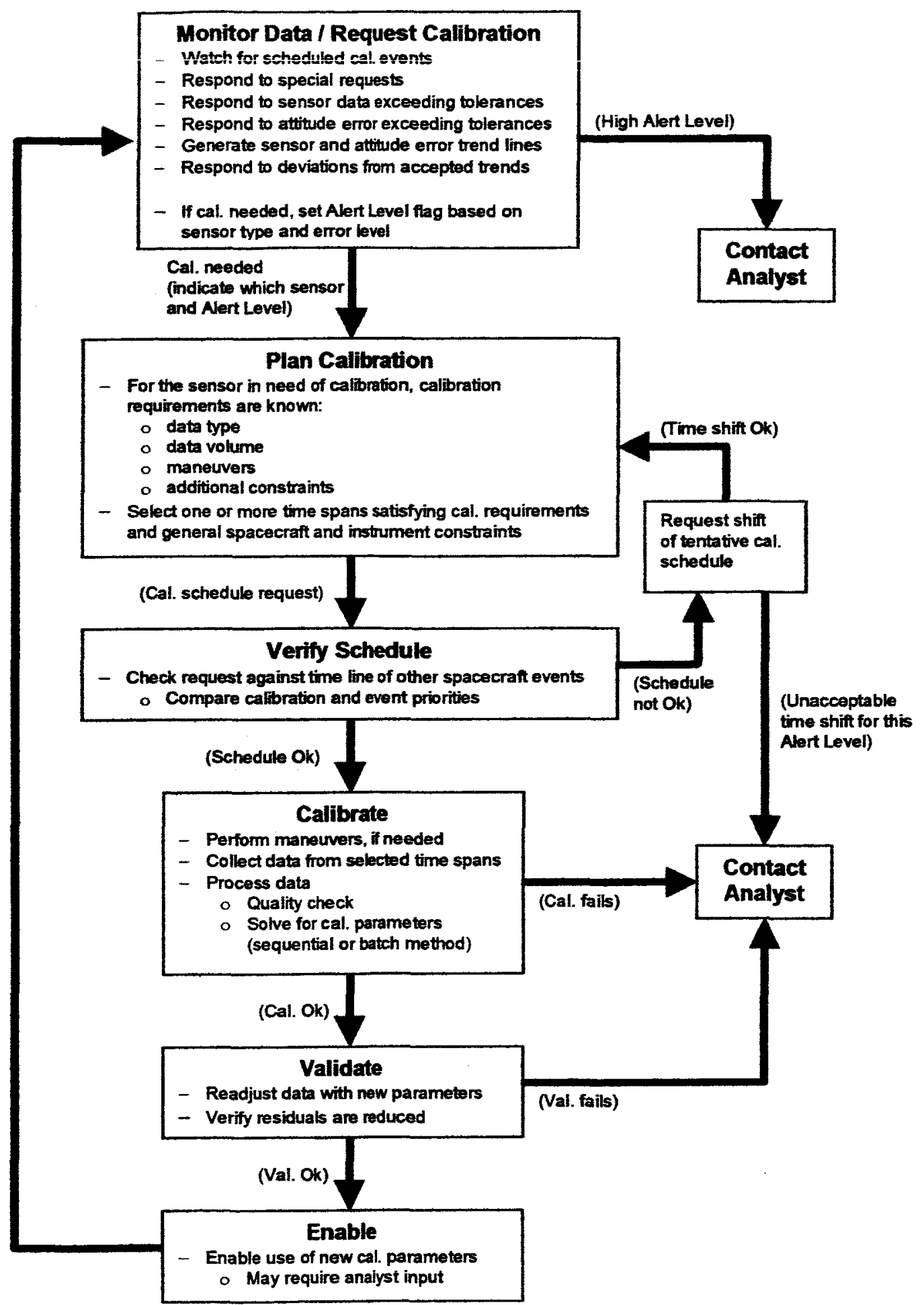

Figure 1. Functional Flow Diagram for Proposed Automated Calibration System. 
Rather than planning maneuvers specifically for IRU calibration, it may be possible to make use of slews already in the science timeline. If the normal mission attitude profile includes rotations that exercise all the gyro axes, no additional calibration maneuvers need to be scheduled. The benefits of this approach can be huge. For missions such as the Hubble Space Telescope (HST) or the Chandra X-ray Observatory, it is very important to avoid interruptions to the observation schedule.

However, to make best use of the maneuvers in the normal mission timeline for IRU calibration, a sequential method is needed. This need arises because a long period of time may elapse before slews on all three axes are available. A batch calibration method needs all the maneuvers at once for processing; whereas, a sequential method can process one maneuver at a time and store any information needed for continuing at a later date (such as the state vector of IRU parameters and its error covariance matrix). Using a sequential method, an automated system can make scheduled calibration requests based on the mission timeline rather than being triggered by large attitude errors after maneuvers. An IRU calibration utility based on a sequential method has been designed and implemented for this purpose. ${ }^{2-3}$ This utility is discussed further in Section III.

The next automated calibration system function is to verify the schedule. The system must check the tentative calibration plan against the mission timeline. Using the Alert Level to set priorities, the tentative schedule can be shifted and replanned until a satisfactory time is chosen.

Next, any required maneuvers are performed and the data are collected, adjusted, and edited to remove outlying points. The appropriate calibration subsystem is called to estimate a set of updated parameters for this sensor or group of sensors.

To validate the new parameters, new data must be processed with both the new and old parameters and used to estimate the spacecraft attitude. If the new parameters are an improvement over the old, the sensor residuals will be statistically smaller when computing a new attitude.

Finally, the use of the new parameters must be enabled. This step may require input from an analyst depending on the level of trust in the automated system. Intervention also may be needed if there are problems with scheduling, large uncertainties reported by the calibration utility, failure to converge, or failure of the validation tests.

\section{IRU Calibration}

The IRU calibration subsystem is the most mature part of the automated calibration system. The subsystem has two main parts: data selection and IRU calibration. The data selection utility includes maneuver detection and selection of time spans for the calibration. It is the newest addition to the automated system. The utility is described in Section III.A. The IRU calibration utility performs the calibration updates, saves required information, and reports results. An early version of the calibration utility was presented in Ref. 2 based on the algorithm given in Ref. 3. This algorithm and some results are briefly reviewed in Sections III.B and C.

IRU calibration consists of estimating improvements to the IRU model parameters. These are the alignments of the 3 gyro sensitive axes, the scale factor for each axis, and the bias for each axis. These 12 independent parameters cannot be distinguished unless the spacecraft performs attitude maneuvers. Thus the first job for the automated system is to locate the maneuvers within a data batch and to select those that are suitable for the calibration. This is the function of the automated data selection utility described next.

\section{A. Automated Data Selection}

One of the most time-consuming steps when performing IRU calibration is selecting time spans holding good attitude data for processing. The calibration algorithm requires accurate attitude solutions before and after each slew and uninterrupted IRU data throughout the time span. The analyst must locate the maneuvers, make allowance for attitude control settling time, estimate the initial and final attitudes, verify attitude accuracy and sensor data quality, and only then perform the calibration. The automated data selection utility performs these steps using a series of tests derived from procedures used by experienced analysts. This utility is designed so that appropriate maneuvers can be selected for a wide range of maneuver types and mission scenarios.

The analyst provides a batch of data holding attitude sensor telemetry in the format used by the attitude ground support system. 'Since the calibration utility uses a sequential algorithm, it is not necessary for the data batch to hold a complete set of maneuvers. For each maneuver the data selection utility finds, it will call the calibration utility to update the estimate of the IRU parameters. Optionally, one of the maneuvers may be a "null maneuver" (that is, a period where the rates remain unchanged) to help distinguish biases from scale factors.

The data selection utility can run either in a fully automated mode or in an interactive mode. The selection process is customized to a particular mission through a set of search and quality check parameters that need to be preset when running in the automated mode. These parameters must be tailored for each specific mission. In the 
interactive mode, all parameters can be modified through graphical user interfaces (GUIs), and the user can override choices made by the data selection procedure.

The test cases given below demonstrate maneuver selections for two very different spacecraft. The RXTE points to several different astronomical targets each day but remains inertially fixed on each target between slews. The IRU calibration uses these maneuvers and other large maneuvers performed specifically for IRU calibration. The Earth Observing System (EOS) Aqua spacecraft rotates at one revolution per orbit (1 rpo) to remain Earth-oriented. A series of moderate sized maneuvers relative to the Earth-pointing frame were performed for IRU calibration.

To detect the start and end of each maneuver, the utility searches for large angular accelerations. It is not enough just to check for changes from the nominal rates since the 1 rpo rotation for an Earth-oriented spacecraft projects onto different axes during a maneuver. For example, the nominal pitch rate becomes a 1 rpo roll rate after a 90 degree yaw. However, searching for accelerations works for both inertial and Earth-oriented spacecraft.

Large angular accelerations are distinguished by comparing each computed acceleration with the standard deviation of the acceleration over the entire time span. The criterion (i.e, how many standard deviations must it be from the norm) is a user input parameter. One complication is that some slews consist of an acceleration, followed by a constant rate coast, and then a deceleration; whereas, other slews have an acceleration and deceleration with no coast period. In either case, the software must recognize the pattern as a single slew.

Figure 2 shows a typical RXTE maneuver. This figure and the following figures in this section show actual onorbit rate data. The upper plot shows rates on the $Z$-axis, and the lower plot shows the accelerations. In this case, the spikes in the acceleration clearly indicate the maneuver start and end times with a long coasting period in between.

A problem arises for spacecraft subject to large vibrations, such as both RXTE and Aqua. The magnitudes of the instantaneous angular accelerations determined by differencing the gyro rates can be as large as typical accelerations for slews. To eliminate these from the maneuver selections, a sliding window average ("boxcar" average) is first applied to smooth the rates. The accelerations are obtained by differencing these smoothed rates. An example is shown in Figure 3. The motion of the massive High-Energy X-ray Timing Experiment (HEXTE) device on RXTE causes significant vibrations twice each minute. The angular accelerations from these vibrations are large even during nominally inertial pointing periods. As seen in the figure, the boxcar averaging reduces the computed accelerations by an order of magnitude so they are not confused with the accelerations from the slews of interest. The width of the smoothing window must be larger than the vibration time but less than the maneuver acceleration time. This window width and all other maneuver detection parameters are adjustable by the user.
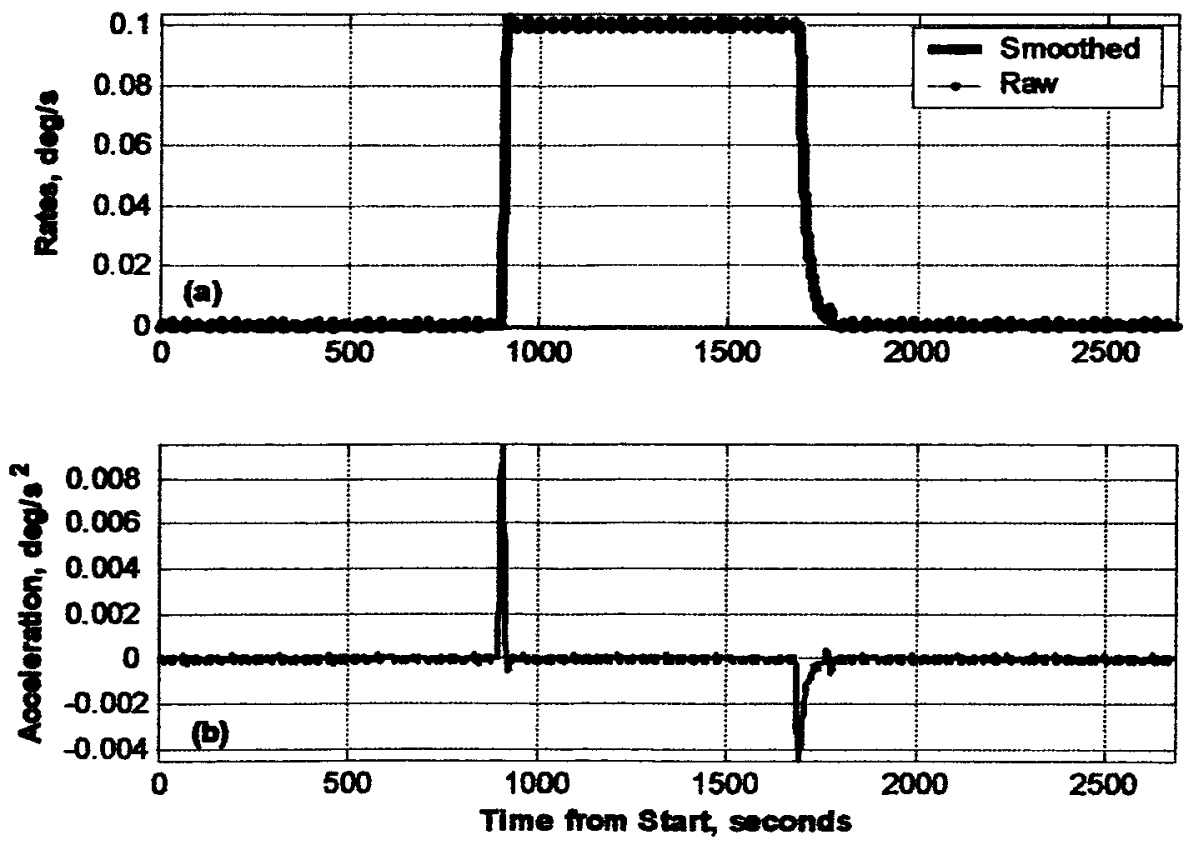

Figure 2. (a) RXTE Z-axis gyro rates for a typical IRU calibration maneuver. The smoothed and raw rates are nearly indistinguishable on this scale. (b) Angular accelerations derived from the smoothed rates. 

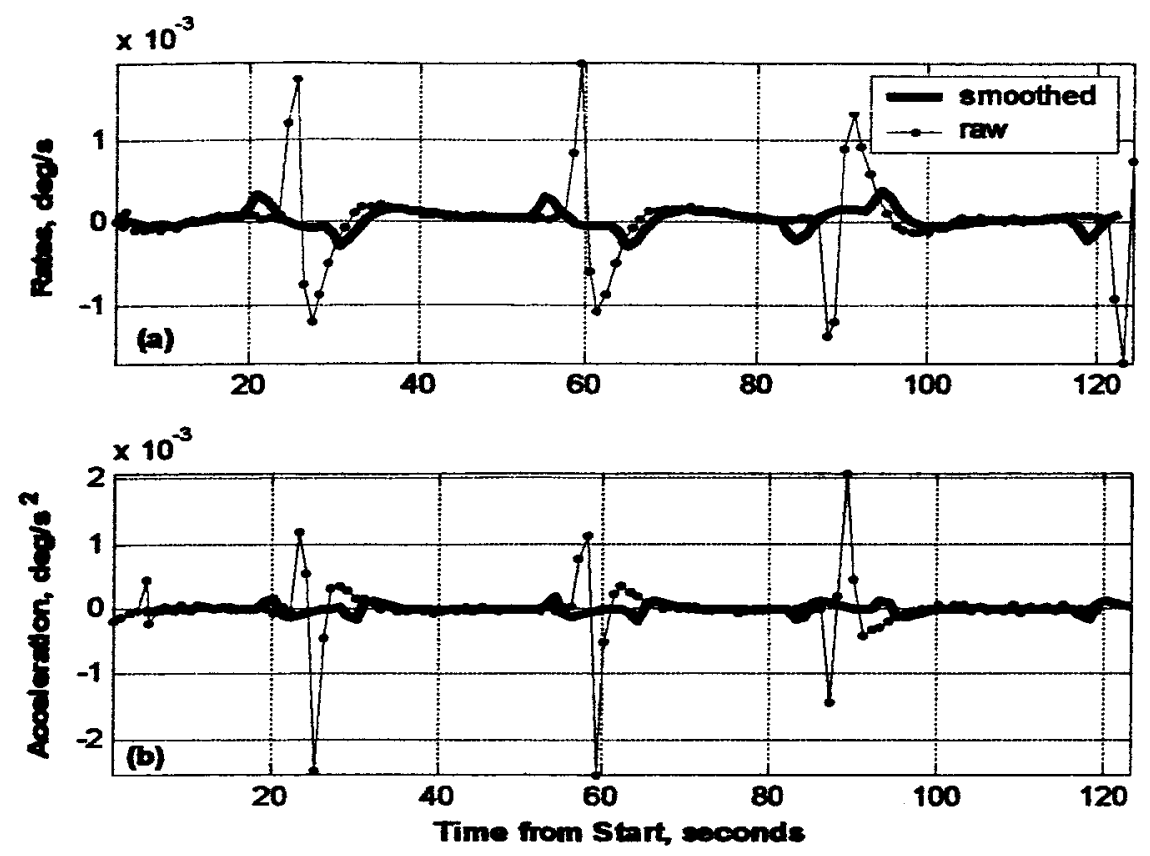

Figure 3. (a) RXTE Z-aris gyro rates for typical inertial period (no maneuver). The large periodic vibrations are caused by motion of one of the science instruments. (b) Angular accelerations derived from the raw (blne) and smoothed (red) rates.

Figure 4 shows typical IRU calibration maneuvers for the Aqua mission. Here there are two slews: the spacecraft pitches up about the Y-axis, remains at that attitude for about 600 seconds, then pitches back to nominal attitude. Each slew consists of an acceleration and deceleration with no coast period. Aqua is an Earth-oriented spacecraft, and the nominal 1 rpo rotation appears as a $-0.06 \mathrm{deg} / \mathrm{s}$ pitch rate on the $Y$-axis.
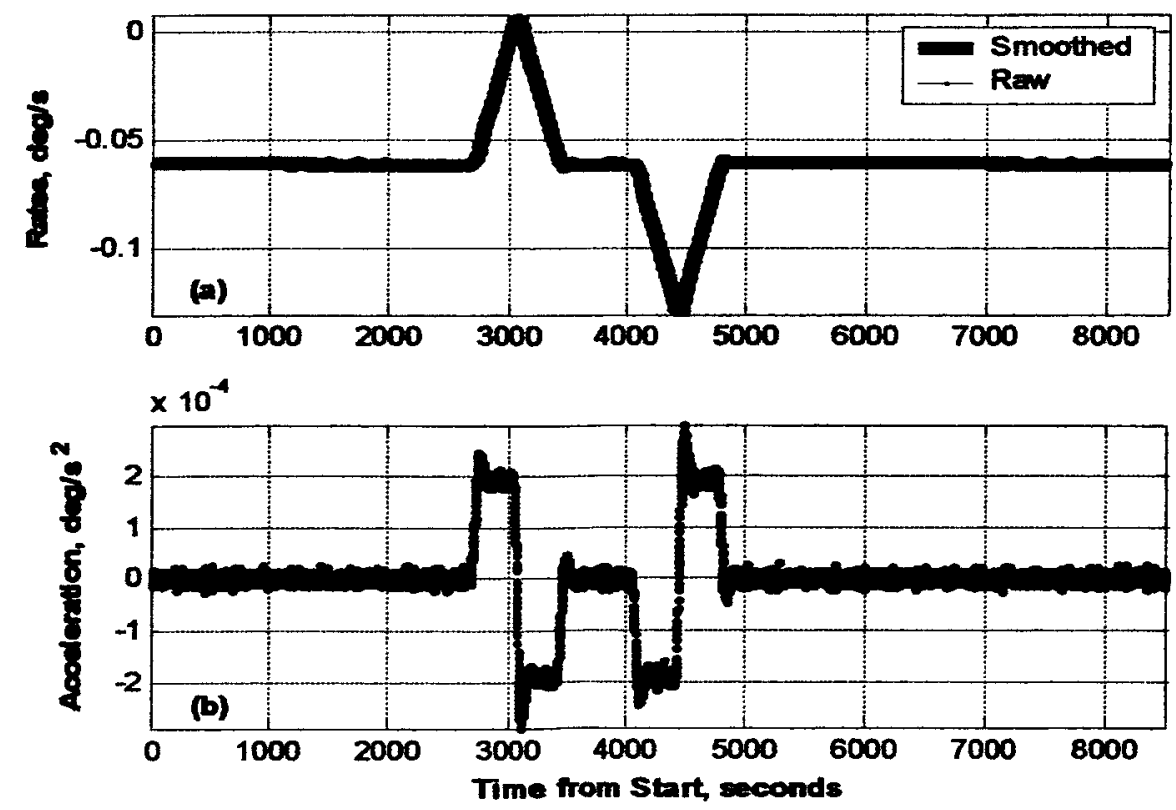

Figure 4. (a) Aqua Y-axis gyro rates for two pitch axis IRU calibration maneuvers consisting of a slew, constant rate hold, and slew back. (b) Angular accelerations derived from the smoothed rates. 
For Aqua, maneuvers about the $\mathrm{X}$-axis are the most difficult to detect. There are large $\mathrm{X}$-axis vibrations due to motion of the science instruments. These vibrations make it hard to determine the roll maneuver start and end. Figure 5 shows the $\mathrm{X}$-axis accelerations after smoothing the rates. The figure shows four roll maneuvers. There is a roll out, constant rate hold for 600 seconds, and roll back. These maneuvers are followed two orbits later by a roll out in the opposite direction, hold, and roll back. These stand out from the vibrations only by about a factor of 3. Without the boxcar smoothing, these slews could not be detected.

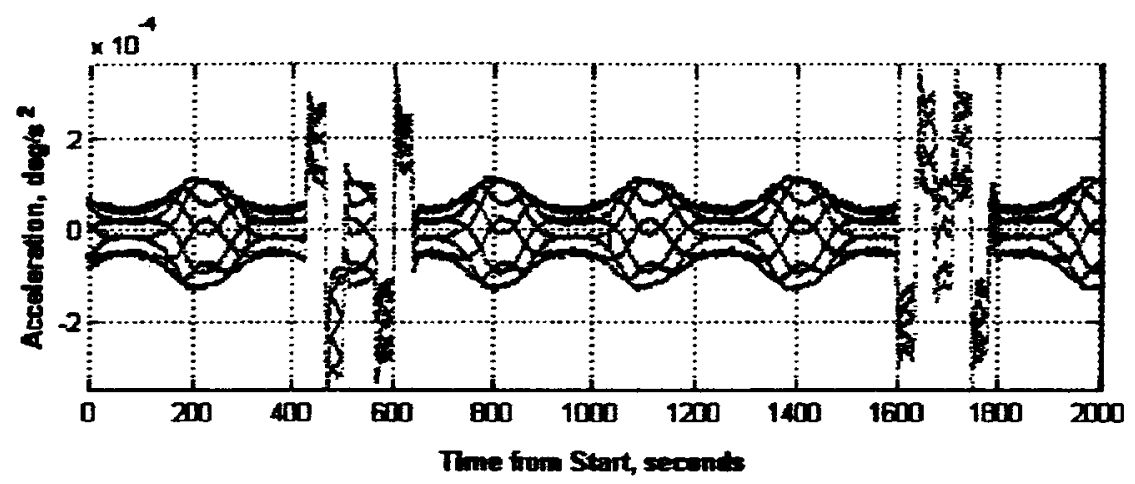

Figure 5. Aqua $X$-axis angular accelerations derived from the smoothed rates. Four roll maneuvers are shown. (The apparent multiple lines are an illusion caused by the IRU sampling frequency relative to the vibration frequency.)

After the maneuvers have been located using the angular accelerations, a user-defined time is subtracted from the start time to allow for broadening of the acceleration period by the smoothing process, and a separate user-defined time is added to the end time to allow for broadening and control system settling at the end.

Next, constant rate periods before and after each maneuver are located and tested for quality. Remember that good attitude solutions will be needed from each constant rate period when performing the calibration.

The following list summarizes the tests that are used to locate data spans with high quality maneuvers for IRU calibration:

1) Angular accelerations are computed from boxcar-averaged gyro rates. Large accelerations and decelerations are paired up as single slews, with separate logic for cases with and without rate coast periods.

2) There must be constant rate periods before and after each slew.

3) The constant rate periods must be sufficiently long.

4) The maneuver must last more than a minimum time. For null maneuvers, the minimum time is taken to be the same as the constant rate period minimum time.

5) The value of the constant rate must be less than a limit. This is needed because sensors (such as star trackers) can have large errors if the angular rates are too large.

6) The standard deviations of the constant rates must be below a given limit.

7) The constant rate periods must have no gyro gaps greater than a given limit.

8) The maneuver periods must have no gyro gaps greater than a given limit.

9) For each sensor used for attitude determination, there must be at least a minimum number of valid observations during the constant rate periods.

If a data span passes the above tests, the attitude determination system (ADS) is run for the two constant rate periods. The ADS is part of the institutional attitude ground support system ${ }^{1}$ and uses a batch least-squares method to estimate the attitude and gyro bias. For each time span, the results of the ADS run must satisfy the following additional tests:

10) The ADS must converge.

11) For each sensor used for attitude determination, the ADS must accept a minimum number of observations.

12) For each sensor used for attitude determination, the ADS must not reject more than a given number of observations. 
13) For each sensor used for attitude determination, the mean of the sensor residuals must be below a given limit.

14) For each sensor used for attitude determination, the standard deviation of the sensor residuals must be below a given limit.

This set of tests is able to detect the significant maneuvers and distinguish them from vibrations and slews that are too small to be useful for IRU calibration. It also ensures that the attitude estimates before and after each slew are sufficiently accurate before being passed to the calibration utility discussed in Section III.B. (The data selection utility does not attempt to check the absolute attitude errors. Instead, the procedure ensures that the errors are typical of the best available with that sensor complement)

The data selection parameters must be adjusted for each mission, but good parameter sets have been found for the very different scenarios posed by RXTE and Aqua, as typified in Figs. 2 through 5.

Figure 6 presents the end result of the data selection utility for a series of several slews for RXTE. In this figure, the black line shows gyro rates, maneuver periods are shaded green, constant rate periods are yellow, and selected constant rate periods are red. For example, the first maneuver is clear since its selected time span does not overlap with the next maneuver. It has nonzero rates on all three axes, and the constant rate periods are indicated before and after the maneuver. The null maneuver can be seen near $1 \times 10^{4}$ seconds. The maneuver near $2.2 \times 10^{4}$ seconds was not selected (there is no red shading) since there were insufficient gyro data before that slew (due to a data dropout in the original telemetry).
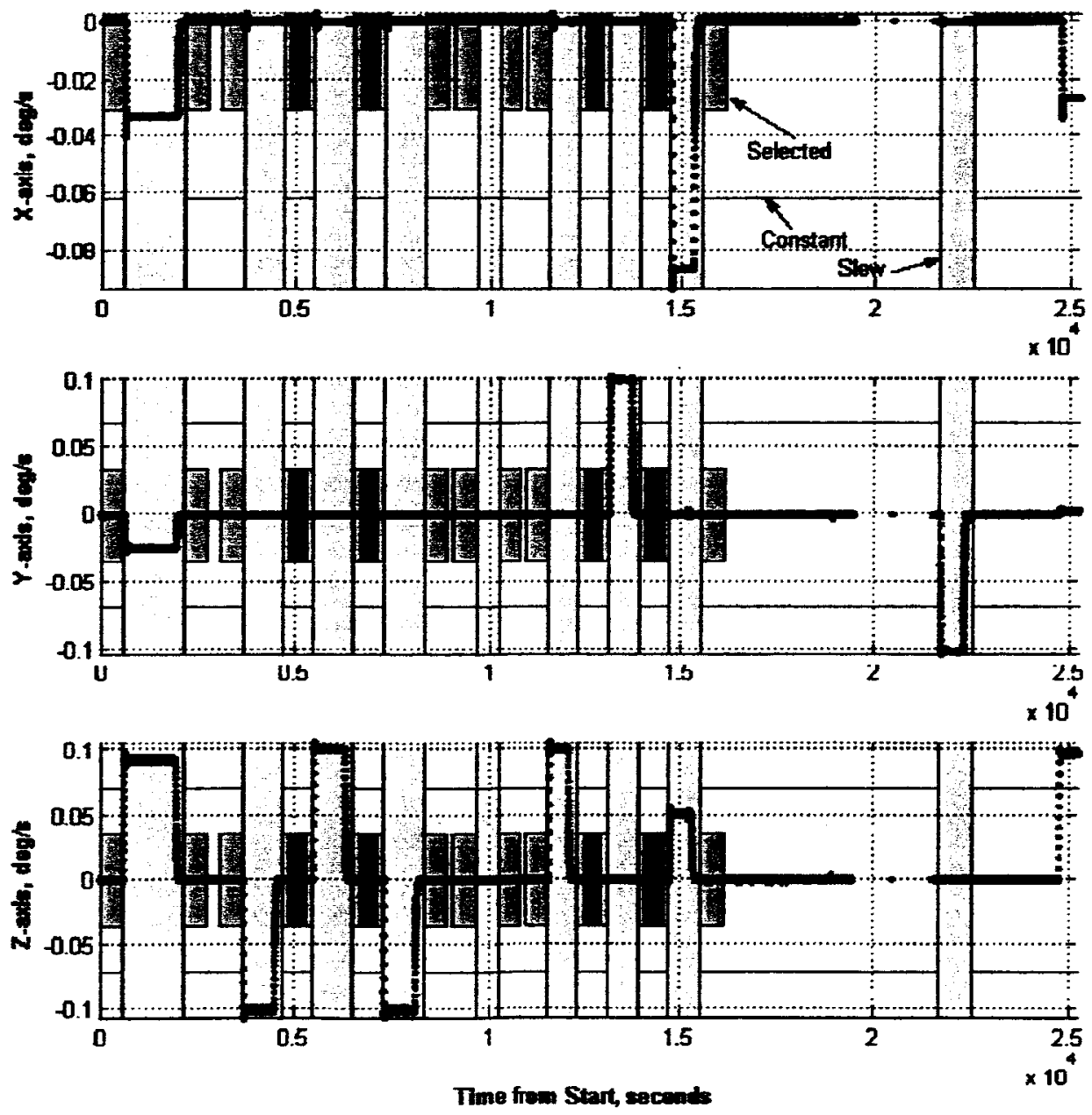

Figure 6. IRU Rates (black dotted line) and data selection for the RXTE spacecraft. The slews are shaded green, the constant rate periods are yellow, and the selected constant rate periods are red. 


\section{B. Sequential Davenport Algorithm}

A variety of IRU calibration methods have been developed and tested in support of missions for NASA/GSFC. Two of the most reliable methods are the Davenport algorithm ${ }^{4}$ and the Batch IRU calibration (BICal) method. ${ }^{5} \mathrm{~A}$ comparison of four different methods is given in Ref. 5 .

The Davenport algorithm has been chosen for the automated IRU calibration utility. The BICal method is simpler to use, but the Davenport method is more robust and more amenable to formulation as a sequential filter.

With a batch method, the entire IRU calibration is performed at one time using a complete set of maneuvers to make all 12 IRU parameters observable. With a sequential method, this is not necessary. The maneuvers can be spread out in time; knowledge of the IRU parameters can be updated after each suitable maneuver. Each slew may contain incomplete information, but complete observability is built up over time as the maneuvers are processed. It is only necessary that the error covariance of the IRU parameters also be updated and carried from one maneuver to the next. This covariance matrix is the means by which correlations among the state elements are remembered and the state can converge when there are sufficient maneuvers for full observability. The method also includes a process noise model to represent the gradual loss of knowledge of the state between maneuvers.

A detailed description of the sequential version of the Davenport algorithm is given in Ref. 3. A review of the theory can also be found in Ref. 2, so only a brief overview will be presented here.

The goal is to determine corrections to the gyro alignments, scale factors, and biases. The raw IRU data are adjusted using a priori values for these 12 parameters to obtain estimated rates, $\vec{\omega}_{0}$. The true rates, $\vec{\omega}$, are unknown.

The difference between the estimated and true rates is a function of the parameter errors. If one has an independent measure of this rate error, the IRU parameter corrections can be estimated.

The attitude estimates during the constant rate periods before and after the maneuvers provide the information needed for an independent measure of the error. The ADS determines both the attitude and an effective bias for each constant rate time interval. This attitude is not affected by the unknown IRU parameter errors. The attitude estimate is accurate because the effective bias absorbs any errors in the 12 IRU parameters as long as the rates are constant. (See the discussion in Ref. 5, for example.) The IRU parameters can only be separately distinguished when the rates change, as during maneuvers. This condition is the reason it is so important to select only data spans where there is good attitude sensor data before and after the slew.

With good attitude estimates in hand, one proceeds by defining an error quatemion

$$
\delta Q=Q_{R} Q_{G}^{-1}
$$

where $Q_{R}$ is a quaternion representing the true vehicle rotation from the start to the end of the maneuver. This total rotation is known, independently of the slew rates, from the ADS attitudes using, e.g., star measurements and the nearly constant rates during each time span. The $Q_{G}$ represents the vehicle rotation inferred entirely from the IRU measurements. One then defines $\delta \vec{z}$ as the vector part of $\delta Q$.

Next, it can be shown that the error $\delta \vec{z}$ is related in $1^{\text {th }}$-order to the angular rate error, $\delta \vec{\omega} \equiv \vec{\omega}-\vec{\omega}_{0}$, through

$$
\delta \vec{z} \approx \frac{1}{2} \int T(t) \delta \vec{\omega} d t
$$

where the matrix $T(t)$ transforms vectors to the pre-maneuver spacecraft frame from the frame of the spacecraft attitude at any time $t$. In particular, $T(t)$ is known from the IRU data throughout the maneuver, adjusted using a priori parameters. The time integration covers the span of the maneuver. (An alternative formulation relates $\delta \vec{z}$ to a standard geocentric inertial frame rather than the pre-maneuver frame, but the end result is the same.)

Equation (2) relates the IRU model parameters to the measurable quantity $\delta \vec{z}$. The error can be expressed in terms of small corrections to the alignment, scale factor, and bias, which can then be estimated by minimizing the error $\delta \vec{z}$ in a least-squares sense.

The weights for the estimator depend on the attitude uncertainties from the ADS and the a priori covariance of the IRU parameters. The algorithm accounts for process noise in both of these terms. A random walk model contributes to the uncertainty both during each maneuver and between maneuvers. The latter is more important because any amount of time may elapse between maneuvers. The strength of the white noise sources underlying the process noise is based on experience with parameter drift from several missions, but filter tuning remains a topic where additional work needs to be done. 
The current implementation of the sequential Davenport algorithm assumes all the gyro data are available within the calibration system so that the solution can be iterated. This helps to remove errors arising from $2^{\text {nd }}$-order terms when the initial errors are large. On the other hand, if the processing were done in real time and the raw IRU data immediately discarded as in an onboard application, it would not be possible to iterate and the convergence time could be longer.

Since the IRU biases may already be known from the ADS solutions during zero rate periods, the calibration utility has an option to use these and to solve only for the alignment and scale factors.

\section{IRU Calibration Results}

The automated IRU calibration subsystem has been tested using flight data and simulations for four different scenarios. Besides the RXTE and Aqua examples discussed above, there are a simulated 1 rpo mission with no noise on the gyros or attitudes and a long duration simulation where the IRU parameters are subject to a random walk. In all cases, the IRU parameters have been corrupted by known amounts, and the errors reported below are relative to these values. The RXTE and Aqua flight data have first been corrected for any prior calibration errors.

\section{Simulated, Noise-Free, 1-rpo Spacecraft}

The first example is a simulated, noise-free, 1 rpo mission. Figure 7 shows the errors in the estimated IRU parameters after each of nine maneuvers. The initial errors in the gyroscope scale factors, alignments, and biases are removed after the first four maneuvers. These are a null maneuver, a 10 degree roll, a -10 degree roll, and a 25 degree yaw. These are followed by a -25 degree yaw, and then a repetition of the roll and yaw offsets in the opposite directions for a total of nine separate slews.

There are three interesting items of note:

First, since this example is noise-free, very little additional improvement in the IRU parameters is obtained with the final five maneuvers.

Second, the errors in the alignment angles get much worse before they get better. Most of this cror can be traced to the large initial bias crrors. In practice, effective biases are determined onboard or on the ground separately from the full IRU calibration, so the initial bias crors can be made much smaller than in this example.
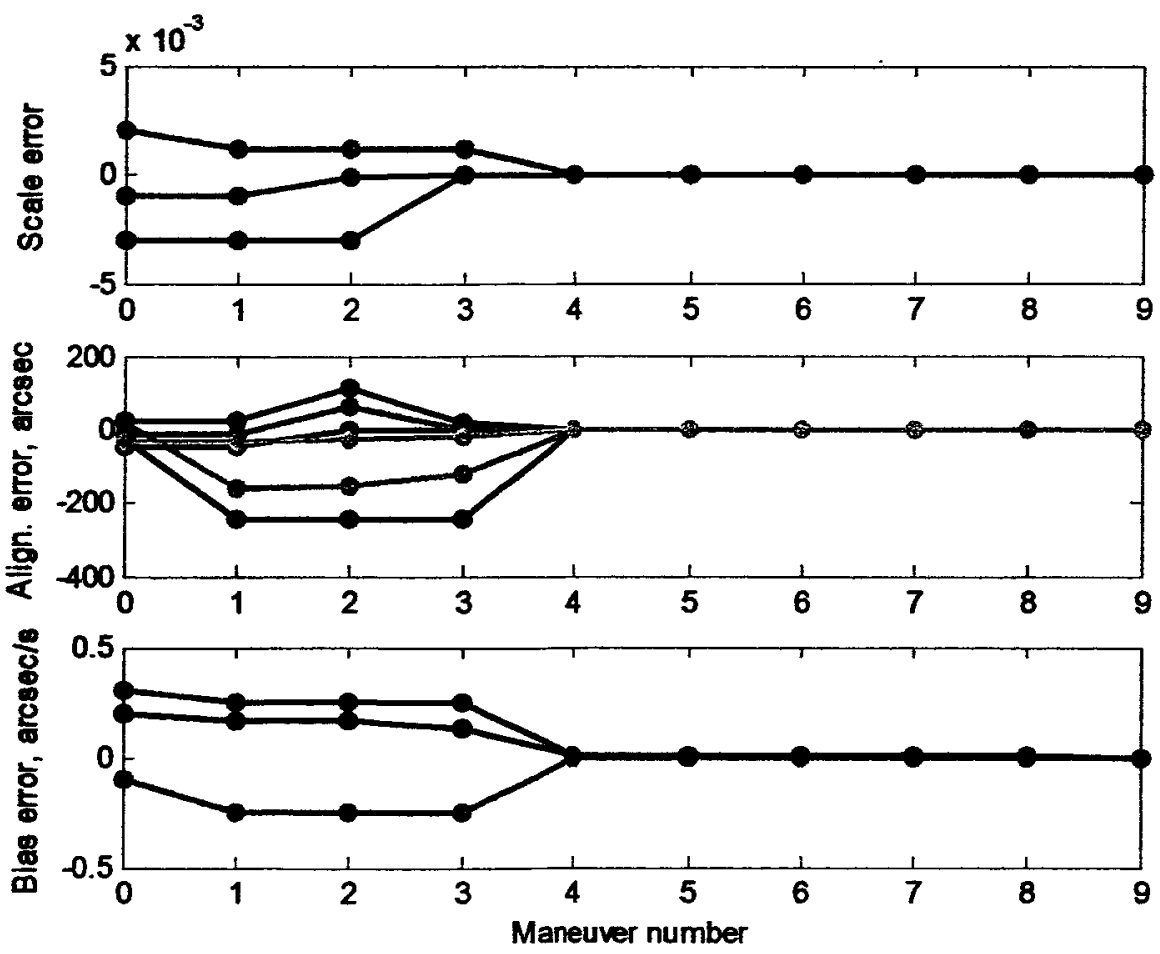

Figure 7. Improvements to the IRU calibration parameters for each maneuver for a simulated, noisefree, Earth-oriented spacecraft. 
Third, no pitch maneuver is needed. Full observability is possible for an Earth-oriented spacecraft using only roll and yaw slews. This condition occurs because the projection of the 1 rpo rotation onto the spacecraft pitch axis is proportional to the cosine of the roll or yaw angle. The change of rotation rate about the body pitch axis is sufficient to make its parameters observable. The fourth scenario given below presents another example similar to this.

For most missions, multiple slews will be performed for IRU calibration, exercising each gyro axis in both directions to provide much more accurate calibration results. The improvement is expected for several reasons: additional data improves signal-to-noise ratio, slews in opposite directions allow for cancellation of some $2^{\text {md }}$-order errors, and some gyros have slightly different scale factors for opposite direction rotations that need to be averaged. Alternatively, the effect of $2^{\text {nd }}$-order errors can be mitigated without slews in opposite directions if the calibration system allows for internal iteration of the state estimate, as is the case with the sequential Davenport algorithm. Also, asymmetric scale factors can be explicitly modeled, increasing the state to 15 elements, but that has not been done in the current implementation.

\section{RXTE Flight Data}

Figure 8 presents an example using actual RXTE flight data after corruptions were added to the IRU rates. The figure shows improvements to the IRU parameter estimates for each maneuver. The calibration was performed with a series of five maneuvers: -80 degrees about the body $Z$-axis, +80 degrees about $Z$, a null maneuver, +60 degrees about $Y$, and 60 degrees about an axis intermediate between $X$ and $Z$. The slew rates in all cases are $0.1 \mathrm{deg} / \mathrm{s}$. The final estimates after five maneuvers are very good. The remaining errors are approximately $2 \times 10^{-6}$ scale factor error, 1 aresec misalignment, and 0.001 aresec/s bias error compared to a prior calibration performed with this same software but with no added corruption.
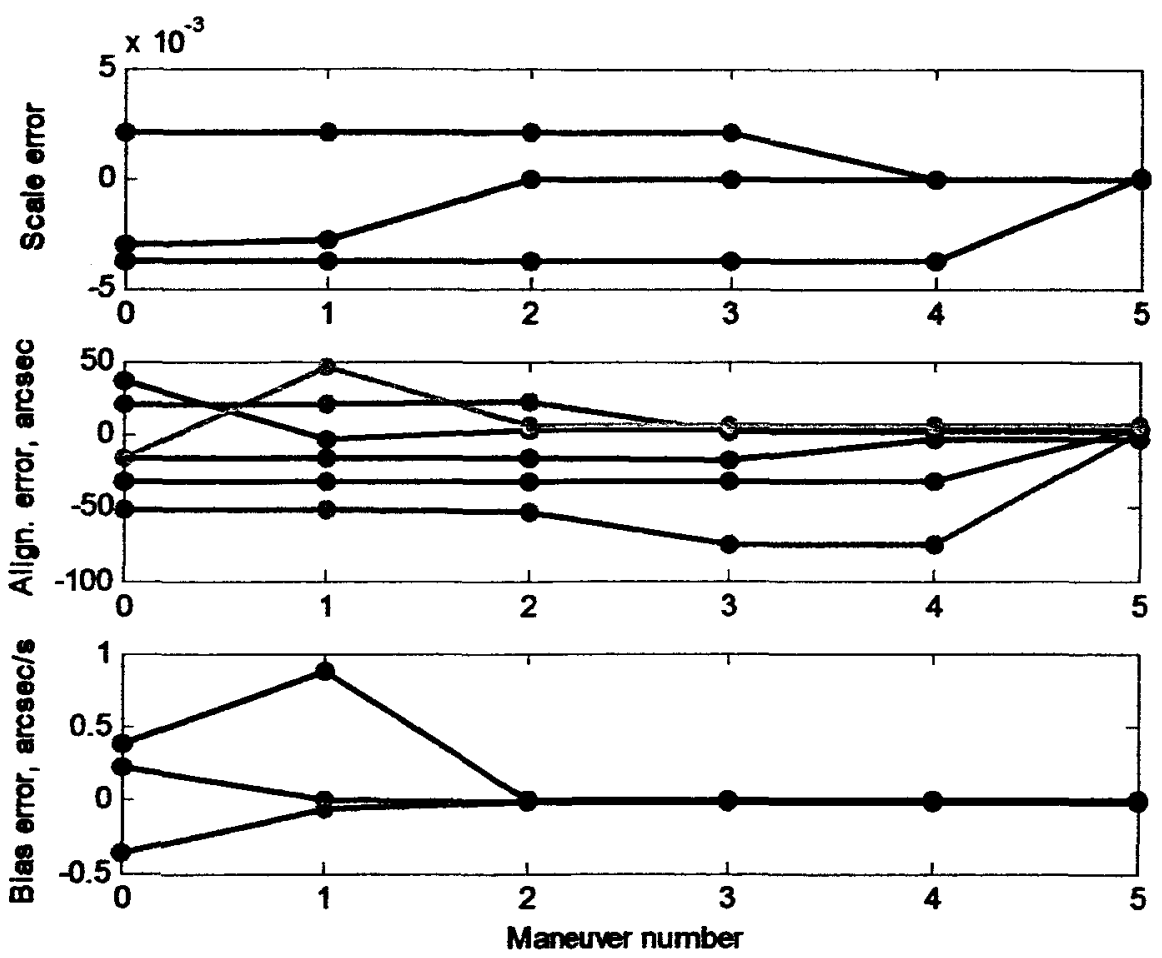

Figure 8. Improvements to the IRU calibration parameters for each maneuver for the RXTE spacecraft asing flight data with corruption added to the IRU parameters.

\section{Simulated RXTE Data, Long Duration, with Random Walk IRU Parameter Errors}

The third scenario is a simulation that consists of an inertially-oriented spacecraft such as RXTE that performs one maneuver per day about a random body axis. It is assumed that the gyro biases are known from the onboard filter, but that the scale factors and alignment drift continually. The goal is to use the one daily maneuver to estimate the scale factors and alignment and to maintain knowledge of them in the presence of the random drift. 
The truth model IRU parameters are modeled as random walks. A random walk produces errors that grow as the square root of the time. For this simulation, the alignment error grows to 3 arcsec and the scale factor error grows to 0.003 in one month. For comparison, this scale factor error is roughly 30 times larger than the tolerance specified for IRUs similar to the ones flown on RXTE, and alignments are usually much more stable than in this example.

Figure 9 shows the results for the first 12 days. The software option not to solve for gyro biases was chosen. The sequential filter locks in on the truth model parameters and successfully follows their drift.

The simulation was continued for five years to verify there were no slowly diverging components. The errors were found to remain small even as the truth model parameters drifted far from their initial values. The standard deviation of the scale factor errors was 0.0007 and the standard deviation of the misalignment was 1.7 arcsec, averaging over the three axes.
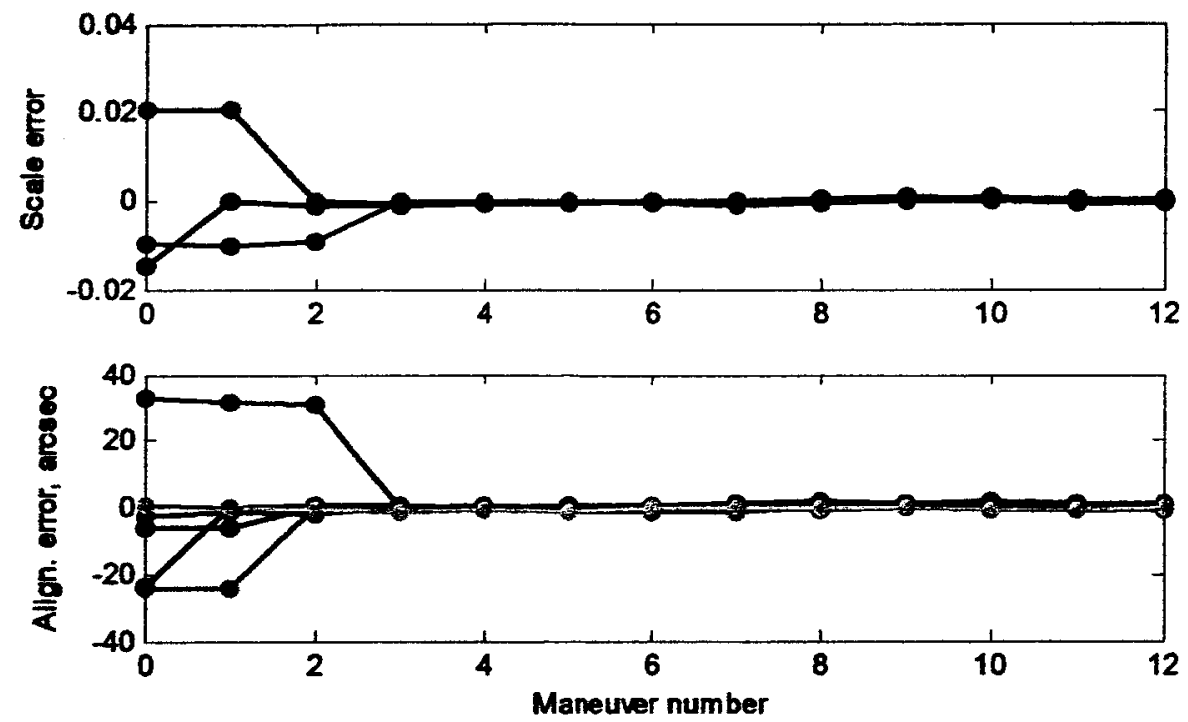

Figure 9. Errors between estimated IRU parameters and truth values for a simulated spacecraft performing one slew per day about a random aris. The calibration converges and parameters remain well determined even with large random walk errors. Biases were not estimated in this test.

\section{Aqua Flight Data}

The final example uses flight data from a series of 12 maneuvers performed over a period of two days by the Aqua spacecraft for IRU calibration. For this test, the raw IRU data were calibrated using the BICal method and then known corruptions were added. There are four maneuvers about each body axis: slew to an attitude offset in roll, pitch, or yaw, hold at that offset long enough to obtain a good attitude solution, slew back to nominal, then repeat with the opposite offset. The $7^{\text {th }}$ maneuver is the null, and what would have been the $13^{\text {th }}$ maneuver was rejected by the data selection utility because of insufficient identified stars. The results are shown in Fig. 10.

It is interesting that a moderately good solution is obtained after only the first four maneuvers. These are all yaw slews. The full state is observable because the yaw rotation exercises the $\mathrm{Z}$-axis gyro, while the yaw offset provides a projection of the 1 rpo rotation rate onto the $\mathrm{X}$-axis proportional to the sine of the yaw angle, and the $\mathrm{Y}$-axis pitch rate decreases from 1 rpo to 1 rpo $\times$ cosine of the yaw angle.

It can also be seen in Fig. 10 that the alignment errors are not completely removed. The final errors range from 10 to 30 arcsec on the three axes. These errors are larger than those for the RXTE example because of uncertainties in the initial calibration. For RXTE, the initial IRU parameter errors were removed using the same data set with the same sequential Davenport software as used for the test, so the end result is expected to be very similar when the corruptions are determined and removed. For Aqua, the BICal utility was used to remove the initial parameter errors. This algorithm is very different in that it uses all the data in the batch rather than selecting only some of the maneuvers and their adjacent constant rate periods. The size of the IRU alignment discrepancy is a measure of the uncertainty in the calibration and is in agreement with previous experience with Aqua. This large uncertainty arises from the large vibrations and time-dependent thermal deformation misalignments of the star trackers discussed briefly in the next section. 

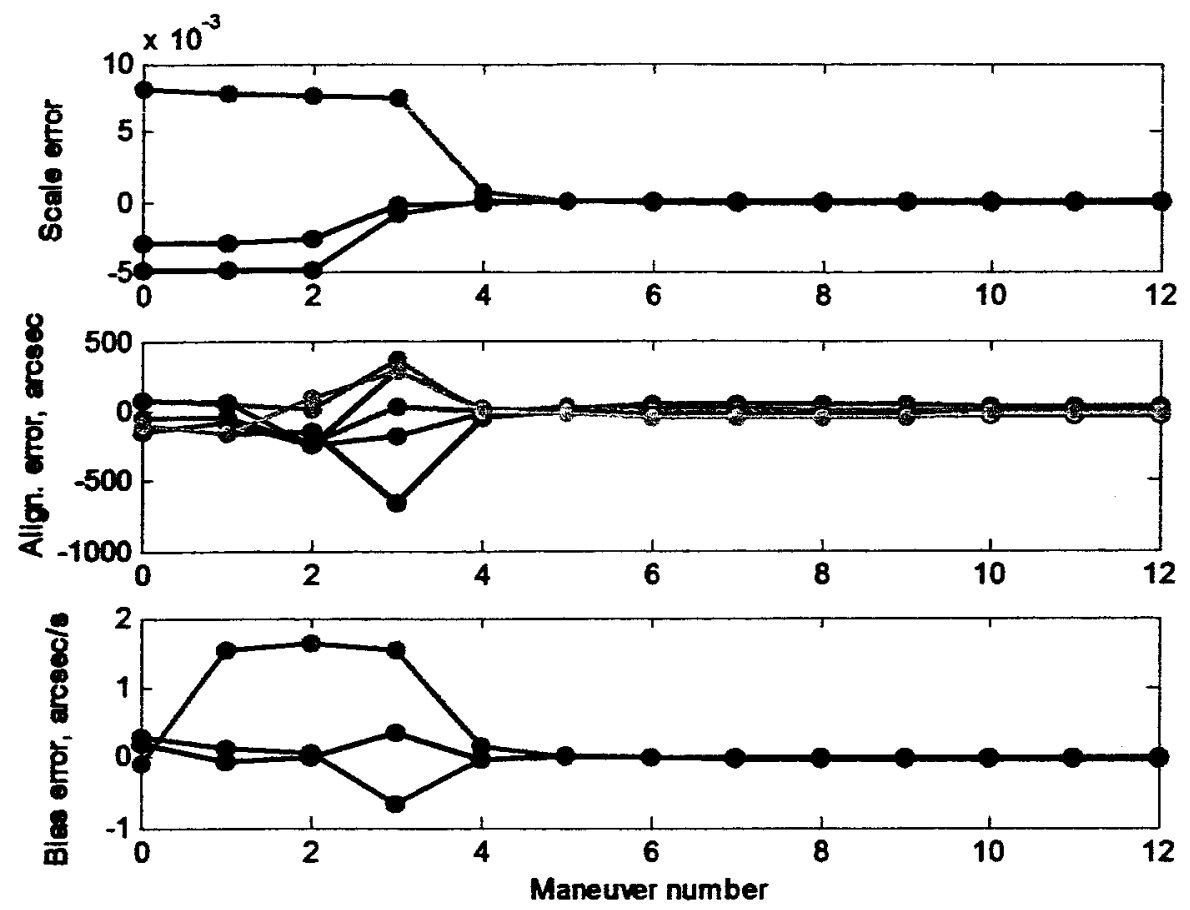

Figure 10. Improvements to the IRU calibration parameters for each maneuver for the Aqua spacecraft using flight data with corruption added to the IRU parameters.

\section{Adtomated Alignment Calibration}

Attitude sensor alignment calibration is a procedure for correcting the sensors for any relative misalignments in their mounting to the spacecraft body. Only relative misalignments can be detected since a common rotation of all the sensors would be indistinguishable from an attitude shif. The alignment calibration procedure is simpler than that for IRU calibration. In addition, it was decided that the initial prototype for automated alignment calibration need not be implemented as a sequential filter. Thus, the design for this subsystem is simpler in many ways than that for IRU calibration.

A reliable, well-tested, batch utility called ALICAL ${ }^{6}$ is available as part of the attitude ground support system for alignment calibration. The ALICAL utility uses an attitude-independent method and is insensitive to rate errors. This also helps simplify the automation process. Many of the requirements for selecting data for the automated IRU calibration subsystem are not needed for alignment calibration.

Similarly to the IRU calibration, the alignment calibration procedure divides into two parts: data selection and alignment calibration. The data selection again is based on finding constant rate periods, but no maneuvers are required. The goal is to locate times when attitude sensor noise is likely to be small. Since sensor error often increases with body rotation rates, the gyro observations are used to determine constant rate periods, and these periods are used for the calibration. The data selection tests are much simpler than those for automated IRU calibration because it is not necessary to locate maneuvers with adjacent constant rate periods.

Once the time span for calibration is selected, the ALICAL utility divides the data into many small groups of nearly simultaneous observations in the sensors to be calibrated. Within each group, the sensor data are all propagated to a common time using the gyro rates. The time length for these groups is set by the user so that the gyro propagation over that time contributes negligible error, which is why the method is insensitive to IRU calibration errors.

Next, ALICAL computes a "single-frame" attitude for each group using the data that were propagated to a common time. These attitudes are used only as a starting point for the ALICAL algorithm. There is no need for any other a priori attitude computation or propagation of attitude estimates between data groups, which is why the method is truly attitude-independent. The program minimizes the root-mean-square sensor residual over all groups with respect to the misalignments.

There are two steps in the process where bad observations may be detected and removed. The first test checks for any observations in each nearly simultaneous group that are inconsistent with the other observations in that 
group. The second test is applied during the iteration process in ALICAL. The program computes the difference between the residuals from each data group and the mean residual for all groups. This difference is compared to the standard deviation of the residuals, and the data group is rejected if the difference is larger than a user-specified tolerance.

A further refinement is required in cases where sensor alignments vary with time and must be frequently recalculated. This was the case for the Aqua mission. Thermal deformations caused the two star tracker alignments to vary regularly as the spacecraft heated and cooled. The deformations were found to repeat approximately with orbit phase. (More accurately, they repeated on every third or fourth orbit due to the combined effects of solar heating and the cycling of an onboard heater.) A mission-specific version of the automated alignment calibration utility has been used to estimate a time-dependent relative misalignment. ${ }^{7}$

\section{Conclusion}

Substantial progress is being made toward the goal of developing an automated calibration system. A functional outline for a very general system has been presented that includes planning and scheduling, as well as calibration and validation. However, the actual prototype code development has focused on the data selection and calibration utilities. A system having just these capabilities should already be useful enough to provide immediate benefits in the operations environment.

The IRU calibration subsystem has been tested with several mission scenarios. It has been found to work well in all cases after the data selection parameters were tailored for each mission. This implies a need to understand the maneuver profile for any mission where this system is to be used. The data selection utility should be tuned using simulated maneuver data before launch.

The sensor alignment calibration subsystem is not yet fully developed. However, an early prototype has been tested with Aqua data and found to work well.

There are a number of areas where additional work is needed. A new subsystem could be developed for automated magnetometer calibration. This utility should be able to process several batches of long data spans. Long data spans are needed to average out the effect of local inaccuracies of the reference field model and to sample a wide variety of magnetic torquer activity so the torquer contamination can be determined.

Another area of interest is onboard automated calibration. In particular, the method used here for IRU calibration can be easily modified for onboard use. In one respect, it would be simpler to apply onboard since there already is an attitude estimate available from the control system. It would not be necessary to determine an attitude for each constant rate period. The utility would only need to select onboard attitude estimates appropriately. Work on such a system is ongoing.

\section{Acknowledgments}

The authors acknowledge the support of the National Aeronautics and Space Administration (NASA) Mission Operations and Missions Services (MOMS) Contract NNG04DA01C, Task Order 088, under which this work was performed. Spacecraft attitude and orbit data were kindly provided by the Flight Dynamics Analysis Branch at NASA/Goddard Space Flight Center, Greenbelt, MD, USA.

\section{References}

${ }^{1}$ Landis, J., a al, "Multimission Threo-Axis Stabilized Spacecraft (MTASS) Flight Dynamics Support System Functional Specifications, Rev. 1," Computer Sciences Corporation technical report prepared for NASAGSFC, 554-FDD-91/070R1UD0, CSCIR-91/6071RIUD0, Sept 1995.

${ }^{2}$ Sedlak, J., Welter, G., and Ottenstein, N., "Towards Automating Spacecraft Attitude Sensor Calibration," 54t Internotional Astronautical Congress, Bremen, Germany, IAF, Sept. 2003.

${ }^{3}$ Welter, G., “A Recursive Filter Approach to Onboard Gyro Calibration (Update 1)," Computer Sciences Corporation technical report prepared for NASAGSFC, CSC-5569-04, Aug. 2003.

'Davenport, P.B., and Welter, G.L., “Algorithm for In-Flight Gyroscope Calibration," Flight Mechanics/Estimation Theory Symposium, CP-3011, NASAGSFC, May 1988.

Hashmall, J., Radomski, M., and Sedlak, J., "On-Obit Calibration of Satellite Gyroscopes," ALAA/AS Astrodynamics Specialist Conference, Denver, CO, Aug 14-17, 2000, AlAA Collection of Technical Papers (A00-39758 10-13).

'Hashmall, J.A, and Sedlak, J.E. "New Attitude Sensor Alignment Calibration Algorithms," 53' International Astronautical Congress, Houston, TX, IAF, Oct. 2002.

Hashmall, J.A., Natanson, G., Gickman, J., and Sedlak, J., “Compensation for Time-Dependent Star Tracker Thermal Deformation on the Aqua Spacecraft," to be presented at the $18^{\text {th }}$ International Symposium on Space Flight Dynamics, Mumich, Germany, Oct. 2004. 